\title{
Pioglitazone Improves Mitochondrial Organization and Bioenergetics in Down Syndrome Cells
}

\begin{abstract}
Nunzia Mollo ${ }^{1}$, Maria Nitti 1,2, Lucrezia Zerillo ${ }^{1}$, Deriggio Faicchia ${ }^{3}$, Teresa Micillo ${ }^{4}$, Rossella Accarino ${ }^{1}$, Agnese Secondo ${ }^{5}$, Tiziana Petrozziello5, Gaetano Cali ${ }^{3}$, Rita Cicatiello ${ }^{1}$, Ferdinando Bonfiglio ${ }^{,}$, Viviana Sarnataro ${ }^{1}$, Rita Genesio ${ }^{1}$, Antonella Izzo ${ }^{1 *}$, Paolo Pinton ${ }^{2}$, Giuseppe Matarese ${ }^{1,3}$, Simona Paladino ${ }^{1}$, Anna Conti ${ }^{1}$ and Lucio Nitsch ${ }^{1,3}$

${ }^{1}$ Department of Molecular Medicine and Medical Biotechnology, University of Naples Federico II, Naples, Italy, ${ }^{2}$ Department of Morphology, Surgery and Experimental Medicine, University of Ferrara, Ferrara, Italy, ${ }^{3}$ Institute of Experimental Endocrinology and Oncology, National Research Council, Naples, Italy, ${ }^{4}$ Department of Biology, University of Naples Federico II, Naples, Italy, ${ }^{5}$ Department of Neuroscience, Reproductive and Odontostomatological Sciences, University of Naples Federico II, Naples, Italy, ${ }^{6}$ Department of Biomedicine, University of Basel, Basel, Switzerland
\end{abstract}

\section{OPEN ACCESS}

Edited by:

Lawrence Todd Reiter,

University of Tennessee Health

Science Center (UTHSC),

United States

Reviewed by:

Alfredo Brusco,

University of Turin, Italy Jorge Busciglio,

University of California, United States

*Correspondence: Antonella Izzo antonella.izzo@unina.it

Specialty section: This article was submitted to Genetic Disorders,

a section of the journal

Frontiers in Genetics

Received: 14 March 2019 Accepted: 07 June 2019

Published: 28 June 2019

Citation:

Mollo N, Nitti M, Zerillo L, Faicchia D, Micillo T, Accarino R, Secondo A, Petrozziello $T$, Cali G, Cicatiello R, Bonfiglio F,

Sarnataro V, Genesio R, Izzo A Pinton P, Matarese G, Paladino S, Conti $A$ and Nitsch L (2019) Pioglitazone Improves Mitochondrial

Organization and Bioenergetics in Down Syndrome Cells.

Front. Genet. 10:606.

doi: 10.3389/fgene.2019.00606
Mitochondrial dysfunction plays a primary role in neurodevelopmental anomalies and neurodegeneration of Down syndrome (DS) subjects. For this reason, targeting mitochondrial key genes, such as PGC-1 $\alpha / P P A R G C 1 A$, is emerging as a good therapeutic approach to attenuate cognitive disability in DS. After demonstrating the efficacy of the biguanide metformin (a PGC-1 $\alpha$ activator) in a cell model of DS, we extended the study to other molecules that regulate the $P G C-1 \alpha$ pathway acting on PPAR genes. We, therefore, treated trisomic fetal fibroblasts with different doses of pioglitazone (PGZ) and evaluated the effects on mitochondrial dynamics and function. Treatment with PGZ significantly increased mRNA and protein levels of PGC-1 $1 \alpha$. Mitochondrial network was fully restored by PGZ administration affecting the fission-fusion mitochondrial machinery. Specifically, optic atrophy 1 (OPA1) and mitofusin 1 (MFN1) were upregulated while dynamin-related protein 1 (DRP1) was downregulated. These effects, together with a significant increase of basal ATP content and oxygen consumption rate, and a significant decrease of reactive oxygen species (ROS) production, provide strong evidence of an overall improvement of mitochondria bioenergetics in trisomic cells. In conclusion, we demonstrate that PGZ is able to improve mitochondrial phenotype even at low concentrations $(0.5 \mu \mathrm{M})$. We also speculate that a combination of drugs that target mitochondrial function might be advantageous, offering potentially higher efficacy and lower individual drug dosage.

Keywords: Down syndrome/therapy, pioglitazone, energy metabolism, oxidative stress, mitochondrial dysfunction, mitochondrial dynamics

\section{INTRODUCTION}

Over the last years, several drugs and nutraceuticals have been tested, mostly in the Ts65Dn mouse model of Down syndrome (DS), aimed at rescuing or attenuating deficits in learning and memory. More than 20 molecules have been successfully identified to restore hippocampal deficits in adult mice (Gardiner, 2015). The results of these preclinical trials suggest that the amelioration or prevention of cognitive deficits in people with DS may be possible, thus paving the way to clinical trials many of 
which are still in progress. Leitmotiv of these investigations was that improving cognitive function would have enormous beneficial consequences for individuals with DS, increasing their potential to participate in society more fully and independently.

In human DS cells we and others have documented an impairment of mitochondrial function with decreased mitochondrial redox activity and decreased levels of mitochondrial electron transport enzymes, altered mitochondrial morphology and increased fragmentation (Piccoli et al., 2013).

Several studies demonstrated that mitochondrial dysfunction plays an important primary role in both altered neurodevelopment and neurodegeneration (Khacho and Slack, 2018). This evidence strongly supports the hypothesis that a mitochondria targeted therapy might attenuate cognitive disability in DS.

Among the molecular mechanisms responsible for mitochondrial dysfunction in DS, we found that the dosage-related over-expression of a corepressor mapping to the chromosome 21 (Hsa21), namely NRIP1, inhibits the activity of the transcriptional activator PGC-1a (Izzo et al., 2014), a gene that orchestrates mitochondrial biogenesis and function (Scarpulla et al., 2012). Indeed, $P G C$ - $1 \alpha$ activity is decreased in cells and tissues of DS individuals (Conti et al., 2007; Piccoli et al., 2013; Izzo et al., 2014), as well as in brain tissues from Alzheimer's disease (AD) patients (Petrozzi et al., 2007), suggesting that similar molecular pathways are involved in $\mathrm{DS}$ and $\mathrm{AD}$ neurodegeneration (Coskun and Busciglio, 2012).

PGC-1 $\alpha$ is a modulator of mitochondrial phenotype, its activity might be targeted through either PGC-1 $\alpha$ activators, successfully tested in mouse models for other diseases (Lagouge et al., 2006; Dong et al., 2007), or agonists of the peroxisome proliferatoractivated receptors (PPARs), which have demonstrated to attenuate mitochondrial dysfunction in $\mathrm{AD}$ mouse models (Nicolakakis et al., 2008; Johri et al., 2012).

We have recently evaluated a PGC-1 $\alpha$ activator, the biguanide metformin, in a DS cell model (Izzo et al., 2017b). Metformin, commonly used in clinical practice in type 2 diabetes as well as in polycystic ovary syndrome and insulin resistance, activates PGC-1a via AMPK and SIRT1 post-translational modifications. We demonstrated that metformin induces both the expression and the activity of PGC-1a in human trisomic fibroblasts, promoting mitochondrial biogenesis (Izzo et al., 2017b).

Even though metformin is a very promising molecule, the use of different drugs acting on same targets through different pathways could be eligible as alternative or synergistic approach. For this reason, in this study, we considered drugs that may regulate mitochondrial function by activating $P G C-1 \alpha$ pathway through the activation of PPARs. Three PPARs have been identified $(\alpha / \gamma / \delta)$, which are ligand-modulated nuclear receptors that regulate gene expression programs of metabolic pathways (Michalik et al., 2006). PPAR- $\gamma$ has proven to be a promising target for treatment of central nervous system diseases. Agonists of this receptor showed to be effective in ameliorating diseaserelated symptoms in animal models (Corona and Duchen, $2015)$. Both PGC-1 $\alpha$ activators and PPAR- $\gamma$ agonists regulate the expression of several target genes involved in neuronal survival and neuroprotection by inhibiting mitochondrial dysfunction, oxidative stress, proteosomal dysfunction, autophagy, neuroinflammation and apoptosis (Chaturvedi and Beal, 2008; Landreth et al., 2008; Corona and Duchen, 2016).

Among the PPAR agonists, pioglitazone (PGZ) (ATC code A10BG03), belonging to thiazolidinediones (TZD) (ATC code A10BG), selectively stimulates PPAR- $\gamma$ (Landreth et al., 2008). PGZ increases the phosphorylation of AMPK and, as consequence, the expression of $P G C-1 \alpha$ and of multiple genes involved in mitochondrial function (Coletta et al., 2009). This drug attenuates mitochondrial dysfunction in animal models of central nervous system injury improving mitochondrial ATP production and oxygen consumption (Nicolakakis et al., 2008; Escribano et al., 2009; Sauerbeck et al., 2011), prevents the loss of dopaminergic neurons (Breidert et al., 2002) and reduces iNOS induction and oxidative stress in mouse models of Parkinson's disease (PD) (Hunter et al., 2008). PGZ is known to normalize hyperglycemiainduced intracellular ROS and mtROS production (Fujisawa et al., 2009). It restores brain ATP levels (Garcia-Bueno et al., 2007) and increases mitochondrial DNA content, oxygen consumption rate (OCR), PGC-1 $\alpha$ and TFAM levels in human adipose tissue and in the neuronal-NT2 cell line (Bogacka et al., 2005; Ghosh et al., 2007). In order to test if PGZ is able to counteract mitochondrial dysfunction in DS, we treated trisomic fetal fibroblasts (DS-HFFs) with different doses of PGZ to evaluate the effects on mitochondrial function and morphology.

\section{MATERIALS AND METHODS}

\section{Ethics Statement}

Four cultures of human fetal fibroblasts, from fetuses with trisomy of Hsa21 at 18-22 gestational weeks, were obtained from the "Telethon Bank of Fetal Biological Samples" at the University of Naples. All experimental protocols were approved by the local Institutional Ethics Committee.

\section{Samples}

Fibroblasts were cultured in T25 flasks (BD Falcon) with Chang medium B $+\mathrm{C}$ (Irvine Scientific) supplemented with $1 \%$ penicillin/streptomycin (Gibco) at $37^{\circ} \mathrm{C}$ in a $5 \% \mathrm{CO}_{2}$ atmosphere. All analyses described throughout this study were carried out at cell culture passages 4-5.

Analysis of karyotype was performed according to standard methods (Genesio et al., 2011).

\section{Pioglitazone Treatment}

PGZ (Cayman Chemical) was dissolved in dimethyl sulfoxide (DMSO) to a stock solution of $5 \mathrm{mM}$ and added to the cell growth medium at the final indicated concentrations. Fresh PGZ was added every $24 \mathrm{~h}$ for $72 \mathrm{~h}$. In untreated control cells an equal volume of DMSO was added every $24 \mathrm{~h}$.

\section{RNA Extraction and Quantitative RT-PCR}

Total RNA from each sample was extracted using TRIzol reagent (Gibco/BRL Life Technologies, Inc., Gaithersburg, MD) and was reverse-transcribed using the iScript cDNA Synthesis kit 
(Bio-Rad Laboratories, Inc., Hercules, CA, USA). Quantitative real time polymerase chain reaction (qRT-PCR) was performed using SsoAdvanced universal SYBR Green supermix on a BioRad iCycler CFX96 Touch Real-Time PCR Detection System according to the manufacturer's protocols. Primer pairs (MWG Biotech, Ebersberg, Germany) were designed using the Primer 3 software (http://bioinfo.ut.ee/primer3-0.4.0/primer3; last accessed date 2015) to obtain amplicons ranging from 100 to 150 base pairs. Primer efficiency was tested generating standard curves for each gene. QRT-PCR results are presented as relative mRNA levels normalized against reference control values. The GAPDH housekeeping gene was chosen as reference gene.

\section{Western Blotting}

Western blotting was performed as previously described (Izzo et al., 2017b). Protein extracts, separated by sodium dodecyl sulphate - polyacrylamide gel electrophoresis (SDS-PAGE) and transferred onto Nitrocellulose membranes, were incubated with the following specific primary antibodies: anti-CASPASE 3 (Cell Signaling Technology), anti-PGC-1 $\alpha$ (Calbiochem), anti-GAPDH (Cell Signaling Technology), anti-Actin (Sigma), anti-MFN1 (Santa Cruz Biotechnology), anti-MFN2 (Santa Cruz Biotechnology), anti-OPA1 (Santa Cruz Biotechnology), and anti-DRP1 (Cell Signaling). Primary antibodies were detected with the appropriate HRP-conjugated secondary antibodies (Santa Cruz Biotechnology or GE-Healthcare) and revealed by chemiluminescence (Pierce) using digital imaging on a Bio-Rad ChemiDoc XRS apparatus or Fuji X-ray film.

\section{Analysis of Mitochondrial Network Architecture}

Cells, seeded at a density of 50,000 per well onto $25-\mathrm{mm}$ glass coverslips, were allowed to grow for $24 \mathrm{~h}$ and then infected with mitochondria-targeted green fluorescent protein (GFP) inserted into an adenoviral vector (Ad-mtGFP Ex/Em: 495/515) as previously described (Izzo et al., 2017b). Protein expression was then allowed for $72 \mathrm{~h}$ in the presence or absence of PGZ.

The efficiency of infection was comparable in treated and untreated trisomic cells both in terms of percentage of GFP positive cells (about 80\%) and of intensity of fluorescent GFP signal.

Single cells were imaged, by using the same settings for treated and untreated cells, with a Nikon Swept Field Confocal microscope (Nikon Instruments Inc.) equipped with a CFI Plan Apo VC60XH objective and an Andor DU885 EM-CCD camera, which was controlled by NIS Elements 3.2. Fiftyone-plane $\mathrm{z}$-stacks were acquired with voxel dimensions of $133 \times 133 \times 200 \mathrm{~nm}(\mathrm{X} \times \mathrm{Y} \times \mathrm{Z})$. The mitochondrial network was then described in numbers of objects, total volume and object volume using the 3D object counter available in the software Fiji (http://www.fiji.sc) (Schindelin et al., 2012). 3D rendering was obtained with the 3D Viewer plugin.

\section{Measurement of ATP by Luciferase Assay}

The cells were plated on 12-mm-diameter glass coverslips for single-sample luminescence measurements and incubated for $24 \mathrm{~h}$. Then they were infected with a VR1012-based construct as previously described (Porcelli et al., 2001) and treated with PGZ or DMSO for further $72 \mathrm{~h}$. Basal ATP content was calculated according to luminescent values of the plateau generated after luciferin addition. Efficiency of luciferase transduction in treated cells and untreated trisomic cells was determined by immunoblot assay as previously described (Izzo et al., 2014).

\section{Measurement of ROS on Single-Cell}

Cells were seeded on glass coverslips and incubated with PGZ or DMSO for $72 \mathrm{~h}$ and then incubated with $2^{\prime}, 7^{\prime}$ dichlorodihydrofluorescein diacetate (DCFH-DA, $17.5 \mu \mathrm{M}$ ) as previously described (Petrozziello et al., 2017). For DCF fluorescence analysis, each coverslip was placed into a perfusion chamber (Medical System, Co. Greenvale, NY, USA) mounted onto a Zeiss Axiovert 200 microscope (Carl Zeiss, Germany) equipped with MicroMax 512BFT cooled CCD camera (Princeton Instruments, Trenton, NJ, USA). Each coverslip was exposed at $485-\mathrm{nm}$ excitation for $10 \mathrm{~s}$ and the emitted light was passed through a 530-nm barrier filter.

\section{Mitochondria Bioenergetics Measurements}

Real-time measurements of OCR were made using an XFe-96 Extracellular Flux Analyzer (Seahorse Bioscience, Billerica, MA, USA). Cells were plated in XFe-96 plates (Seahorse Bioscience) at the concentration of 25,000 cells/well. Cells were counted before and after the experiments. OCR was measured in XFe media (non-buffered Dulbecco's modified eagle medium (DMEM) medium containing $10 \mathrm{mM}$ glucose, $2 \mathrm{mM}$ L-glutamine and $1 \mathrm{mM}$ sodium pyruvate) under basal conditions and in response to 5 $\mu \mathrm{M}$ oligomycin, $1.5 \mu \mathrm{M}$ of carbonyl cyanide-4-(trifluoromethoxy) phenylhydrazone (FCCP) and $1 \mu \mathrm{M}$ of Antimycin-A and Rotenone (all from Sigma-Aldrich) as previously described (Izzo et al., 2017b). Each sample was plated at least in triplicate.

\section{Statistical Procedures}

Unless otherwise indicated, all assays were performed independently and in triplicate. Statistical analysis was performed using GraphPad Prism software vers.5.0 (GraphPad Software, La Jolla California USA, http://www.graphpad. com). Student's t test was applied to evaluate the statistical significance of differences measured throughout the data sets presented. The threshold for statistical significance ( $\mathrm{p}$-value) was set at 0.05 .

\section{RESULTS}

In order to understand the impact of PGZ on mitochondrial function we exposed DS-HFF cells to different concentrations of the drug for $72 \mathrm{~h}$. According to PGZ half maximal effective concentration $\left[\mathrm{EC}_{50}=\sim 500-600 \mathrm{nM}\right.$ for both human and murine PPAR- $\gamma$ activation (Sakamoto et al., 2000; Willson et al., 2000)] 
and previous reports in which the effects of others TZDs were analyzed (Hofer et al., 2014), we tested four increasing PGZ concentrations between 0.25 and $2 \mu \mathrm{M}$ in DS-HFFs. Higher doses were not tested because they exert inhibitory effects on the mitochondrial respiratory chain (Garcia-Ruiz et al., 2013).

\section{PGZ is Not Toxic in Trisomic Cells and Induces PGC-1 $\alpha$ Expression}

To evaluate the potential toxicity of PGZ in trisomic cells, we evaluated the cell growth by MTT assay, the live/dead cell viability using the Trypan Blue dye exclusion test and the caspase activity by Western blot after $72 \mathrm{~h}$ of treatment at the intermediate and the highest concentrations $(0.5$ and $2 \mu \mathrm{M})$.

MTT assay is a sensitive indicator of the cellular metabolic activity being dependent on mitochondrial respiration (Mosmann, 1983). No significant variation in cell viability was found after exposure to the drug at both concentrations in comparison with trisomic cells treated with solvent only (Figure S1A). To exactly determine the number of viable cells after treatment, we used Trypan Blue dye. We found that the percentage of dead cells for each condition was around 2\% in PGZ treated trisomic cells and controls, indicating that PGZ does not induce cellular death (Figure S1B). In addition, we evaluated in these cells the activity of Caspase 3, one of the most relevant biochemical markers of cell death. We did not find cleavage of caspase 3 in any of the conditions (Figure S1C). PGZ significantly increased the PGC-1 $\alpha$ protein levels starting from $0.25 \mu \mathrm{M}$ (Figure 1A, B). Consistently, $P G C-1 \alpha$ mRNA levels were significantly increased in trisomic cells after PGZ treatment (Figure 1C).

\section{PGZ Affects the Mitochondrial Network Architecture by Modulating the Fission/ Fusion Machinery}

To gauge if PGZ affects mitochondrial morphology in trisomic cells, we expressed a mitochondria-targeted GFP in DS-HFFs and measured mitochondrial number and mitochondrial volume in PGZ-treated versus untreated cells. At confocal microscopy analysis PGZ-treated cells exhibited a less fragmented mitochondrial network with elongated and branched mitochondria (Figure 2B, C) when compared with untreated trisomic cells (Figure 2A), thus resembling to those observed in euploid cells (Izzo et al., 2017b). In detail, treated trisomic cells showed on average a lower number of mitochondria (Figure 2D) and an increase of individual mitochondrial volume (Figure 2F) when compared with untreated trisomic cells. Total volume was significantly increased only at $2 \mu \mathrm{M}$ PGZ concentration (Figure 2E). Overall mitochondrial network was restored by PGZ administration in a dose-dependent manner.

To further investigate molecular bases of the increased connectivity mediated by PGZ, the expression level of genes responsible for mitochondrial fusion (OPA1, MFN1, and MFN2) and fission (DRP1) was assessed. The treatment with PGZ significantly upregulated OPA 1 expression at both 0.5 and $2 \mu \mathrm{M}$ concentrations and MFN1 expression at $2 \mu \mathrm{M}$ concentration

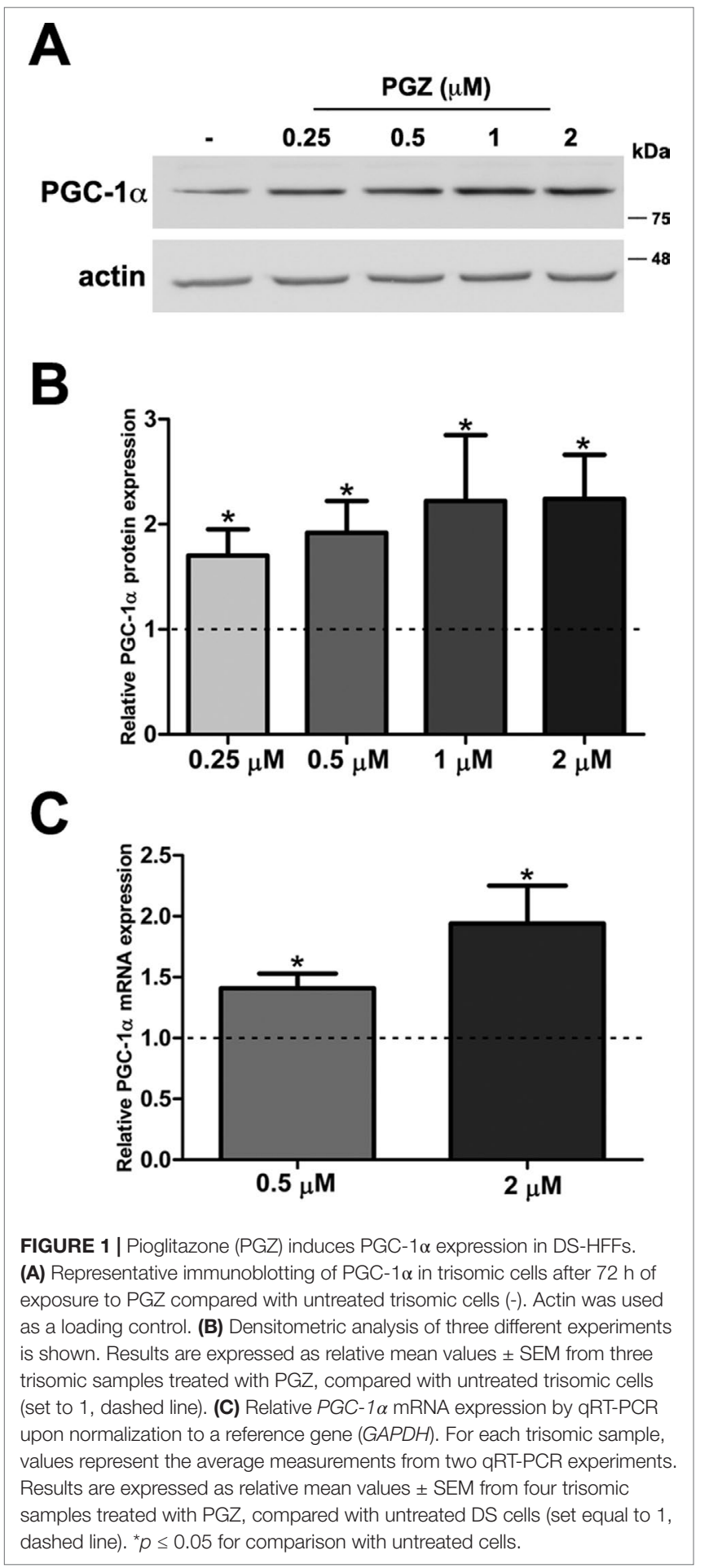

(Figure 3A). No variation of MFN2 gene expression was found, while the mRNA of DRP1 was significantly decreased.

At the protein level, only OPA1 expression was significantly increased in treated cells (Figure 3B). Neither mitofusins nor DRP1 showed significant variation at the protein level (Figure 3C-E). 

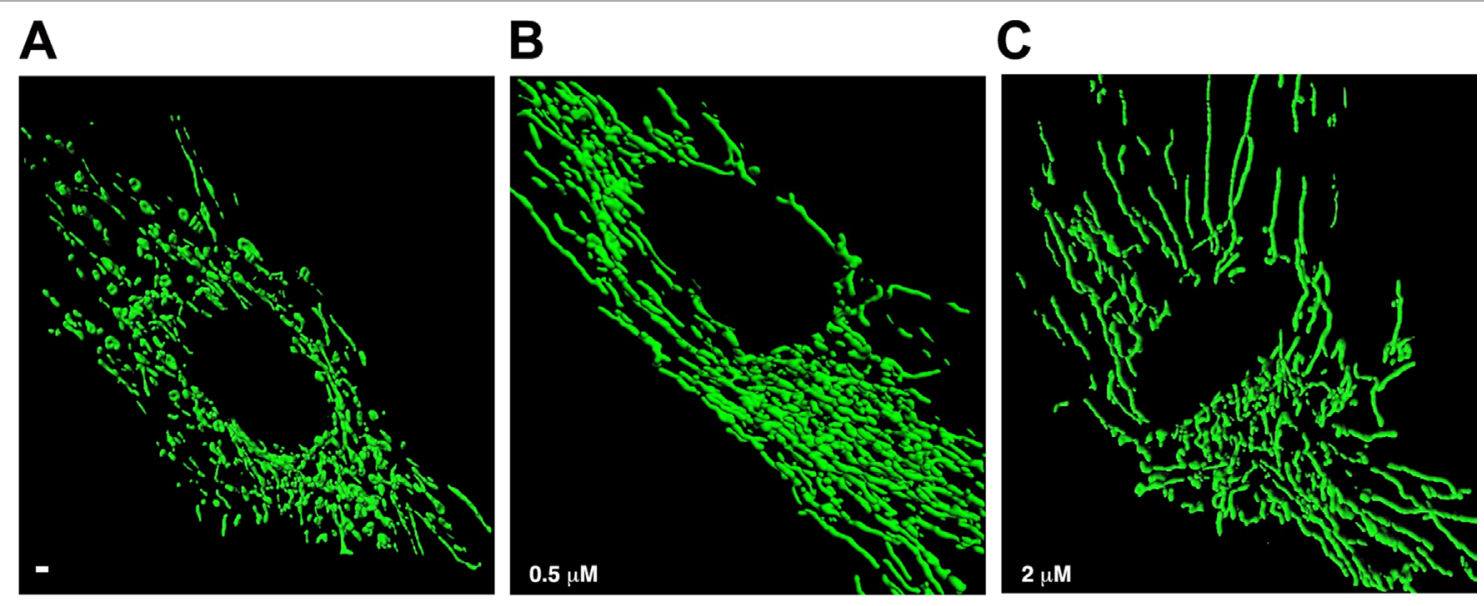

D

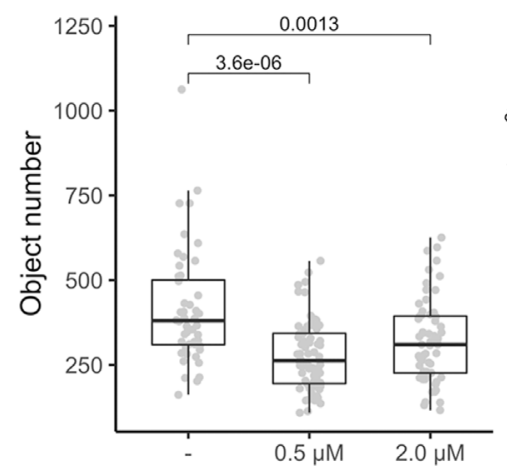

E

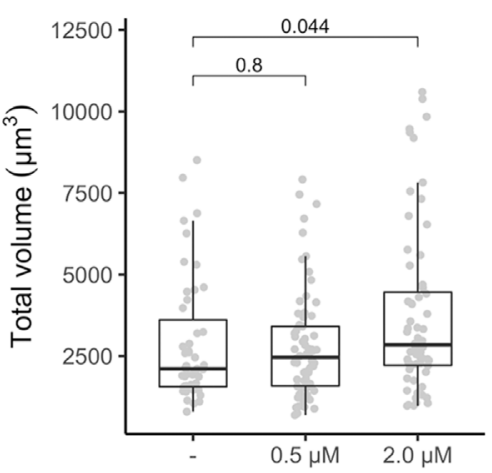

$\mathbf{F}$

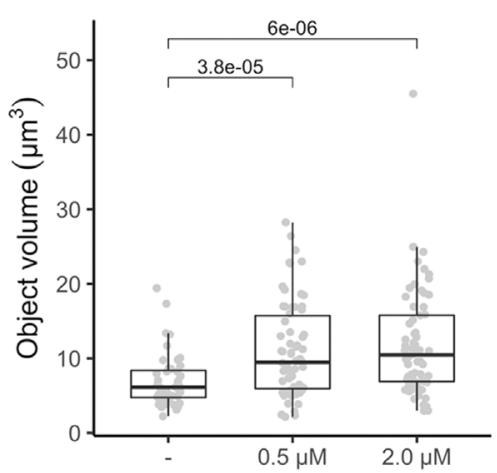

FIGURE 2 | Pioglitazone restores mitochondrial network organization in DS-HFFs. Representative images of the mitochondrial network in (A) untreated (-) and trisomic cells treated with (B) $0.5 \mu \mathrm{M}$ and (C) $2 \mu \mathrm{M}$ PGZ. 3D reconstruction of Z planes acquired by using the same settings with voxel dimensions of $133 \times$ $133 \times 200 \mathrm{~nm}(X \times Y \times Z)$. Box and whisker plots represent the number of mitochondria (D), the mitochondrial total volume (E) and the individual mitochondrial volume (F) measured in PGZ-treated trisomic cells and untreated cells (-). The plots show measurements from fifty randomly selected cells from four samples analyzed in each experimental condition. P-values of comparison between untreated and treated cells are indicated on plots.

\section{Bioenergetics of Trisomic Cells Is Improved by PGZ Treatment}

Since ATP content was previously found to be decreased in trisomic cells (Izzo et al., 2017b), using a mitochondria targeted luciferase we measured the ATP content in treated and untreated trisomic cells. A 60\% increase in basal ATP content, calculated by the luminescence values of the plateau generated after the addition of luciferin, was detected in trisomic cells treated at 0.5 and $2 \mu \mathrm{M}$ PGZ doses (Figure 4A). The luciferase transduction, determined by immunoblot assay, was comparable between treated and untreated trisomic cells (data not shown).

As trisomic cells display an increased ROS production with a larger redox imbalance when compared with euploid cells (Piccoli et al., 2013), we investigated the effects of PGZ on intracellular ROS in trisomic cells using the redox-sensitive probe DCFH-DA. Since ROS measurement by DCFH is affected by the duration and intensity of light excitation, the time of exposure at $485 \mathrm{~nm}$ was fixed at $10 \mathrm{~s}$ for all the samples.
DCF associated fluorescence was significantly decreased in trisomic cells after treatment with 0.5 and $2 \mu \mathrm{M}$ PGZ for $72 \mathrm{~h}$ in a dose-dependent fashion (Figure 4B).

All together these results demonstrated an increase of mitochondrial bioenergetics in treated cells, which is significant already at low concentration of the drug. As confirmation of these data we directly measured Basal OCR, ATP-linked respiration, and maximal respiration by XFe-96 Extracellular Flux Analyzer (Seahorse Bioscience). Cells exposed to $0.5 \mu \mathrm{M}$ PGZ showed significant increase of all the parameters when compared with untreated cells (Figure 5A, B).

\section{DISCUSSION}

Although the phenotype of DS is complex, intellectual disability and an early development of AD neuropathology occur in almost all the individuals affected by DS (Gardiner, 2015). Increasing evidence indicates that mitochondrial dysfunction, observed 

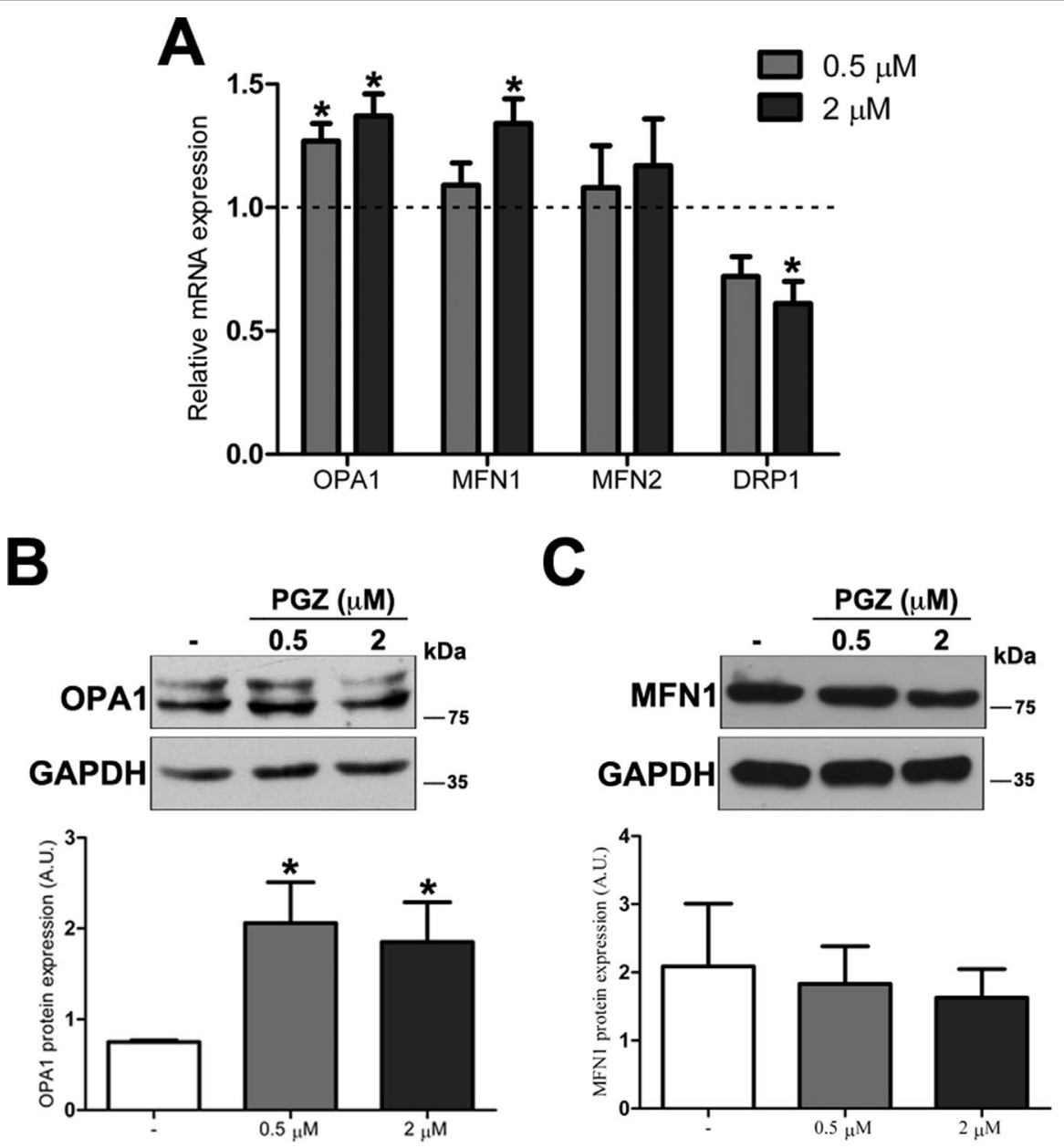

D

$\mathbf{E}$
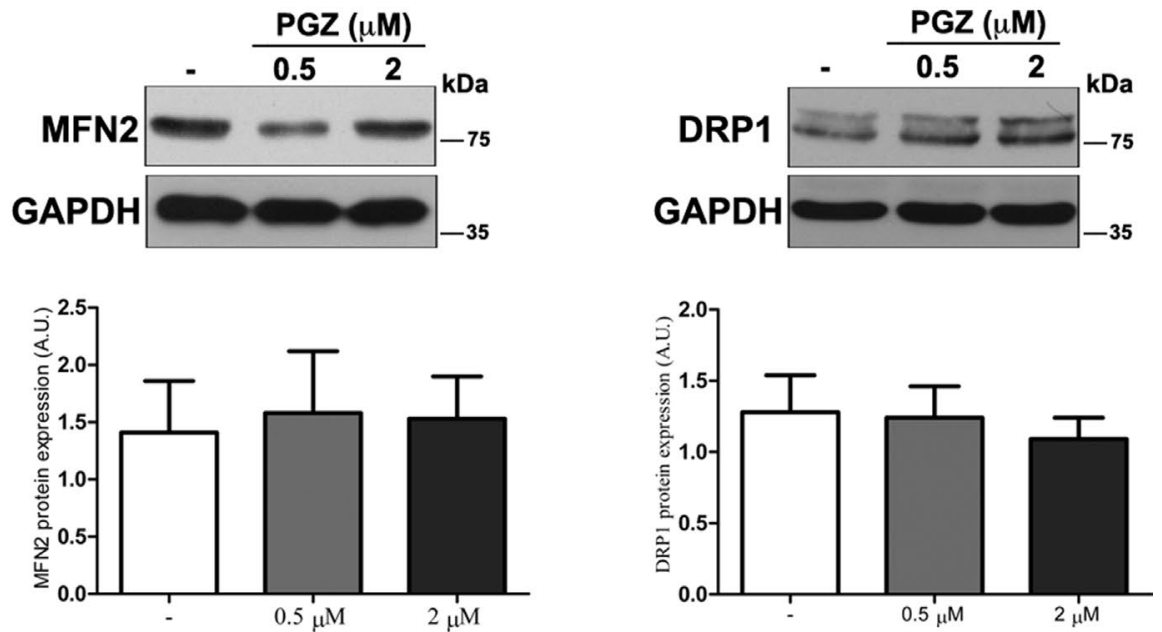

FIGURE 3 | Pioglitazone modulates the expression of mitochondrial fission-fusion genes in DS-HFFs. (A) Relative OPA1, MFN1, MFN2, and DRP1 mRNA expression after $72 \mathrm{~h}$ of treatment with PGZ $(0.5$ and $2 \mu \mathrm{M})$ measured by qRT-PCR upon normalization to a reference gene $(G A P D H)$. For each trisomic sample, values represent the average determination for 2 qRT-PCR experiments. Results are expressed as relative mean values \pm SEM of cell cultures from four trisomic samples treated with PGZ, compared with untreated DS cells (set equal to 1 = dashed line). Representative immunoblotting of (B) OPA1, (C) MFN1, (D) MFN2, and (E) DRP1 after $72 \mathrm{~h}$ of treatment with PGZ and densitometric analysis of four different experiments are shown. GAPDH was measured as a loading control. Results are expressed as mean values \pm SEM from trisomic samples treated with PGZ and from untreated cells. ${ }^{*} p \leq 0.05$ for comparison with untreated cells. 


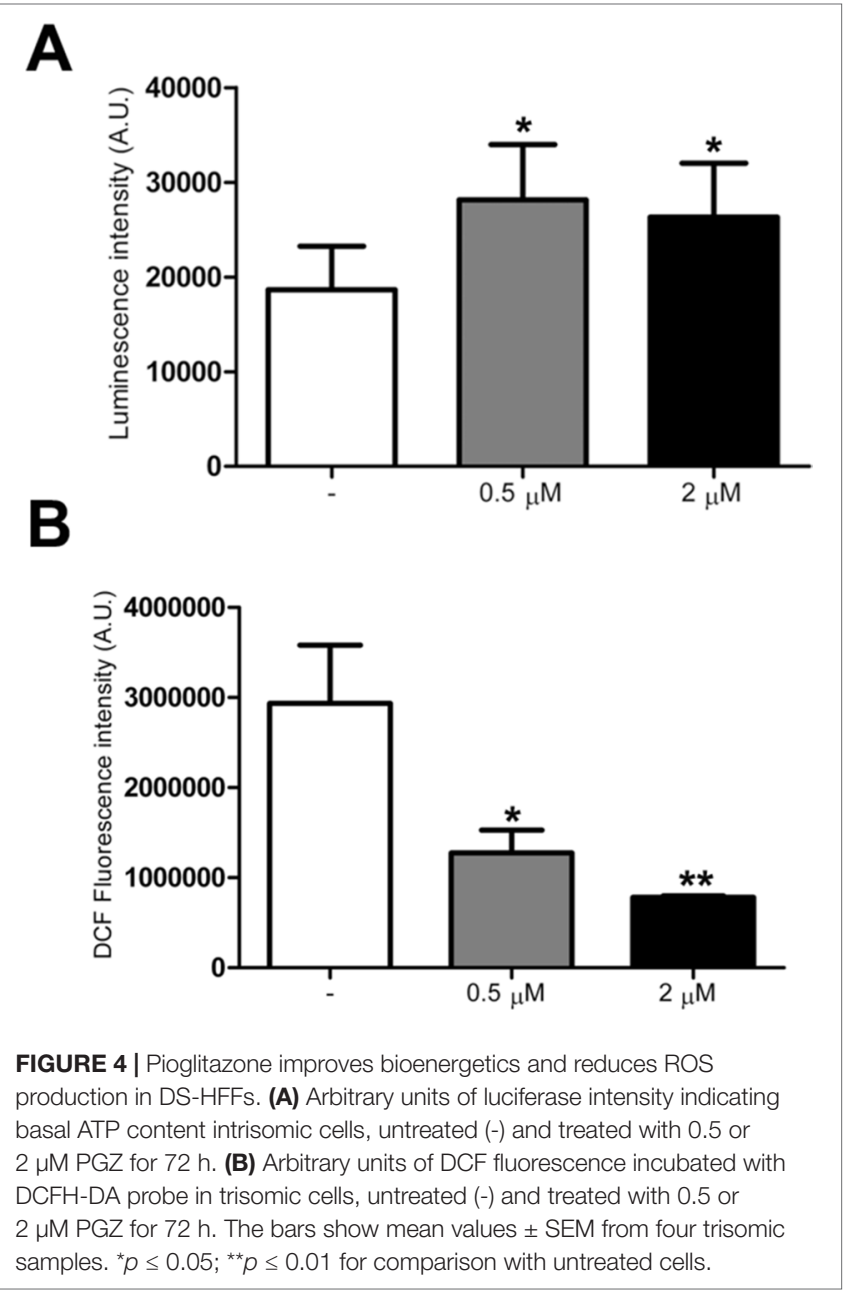

in DS, contributes to generating these phenotypic traits, as mitochondrial dynamics and metabolism play an important role in both fetal and adult neurogenesis (Beckervordersandforth et al., 2017; Khacho and Slack, 2018). Accordingly, DS is associated with impairment in several structural/cognitive domains. Fetuses with DS exhibit a reduced number of neurons and a relative increase in the number of astrocytes. The brains of fetuses with DS show weight (Guihard-Costa et al., 2006) and volume reduction in various hippocampal structures (Guidi et al., 2008) and in the cerebellum (Guidi et al., 2011). Developmental defects in the dentate gyrus, altered dendritic spine morphology and reduced spine and synaptic density have also been observed (Guidi et al., 2018). These last features have been associated with the astrocytesecreted thrombospondin 1 (Torres et al., 2018). Recent studies also demonstrated an important role of PGC-1 $\alpha$ and of its targets in the formation and maintenance of hippocampal dendritic spines and synapses (Cheng et al., 2012). Impaired PGC-1 $\alpha$ activity is emerging as a common underlying cause of mitochondrial dysfunction in neurodegenerative diseases such as $\mathrm{AD}, \mathrm{PD}$ and Huntington disease (Correira, 2018). Pgc-1 $\alpha(-/-)$ mice showed a decreased mitochondrial number and respiratory capacity and vacuolar lesions in the central nervous system (Leone et al., 2005). All these

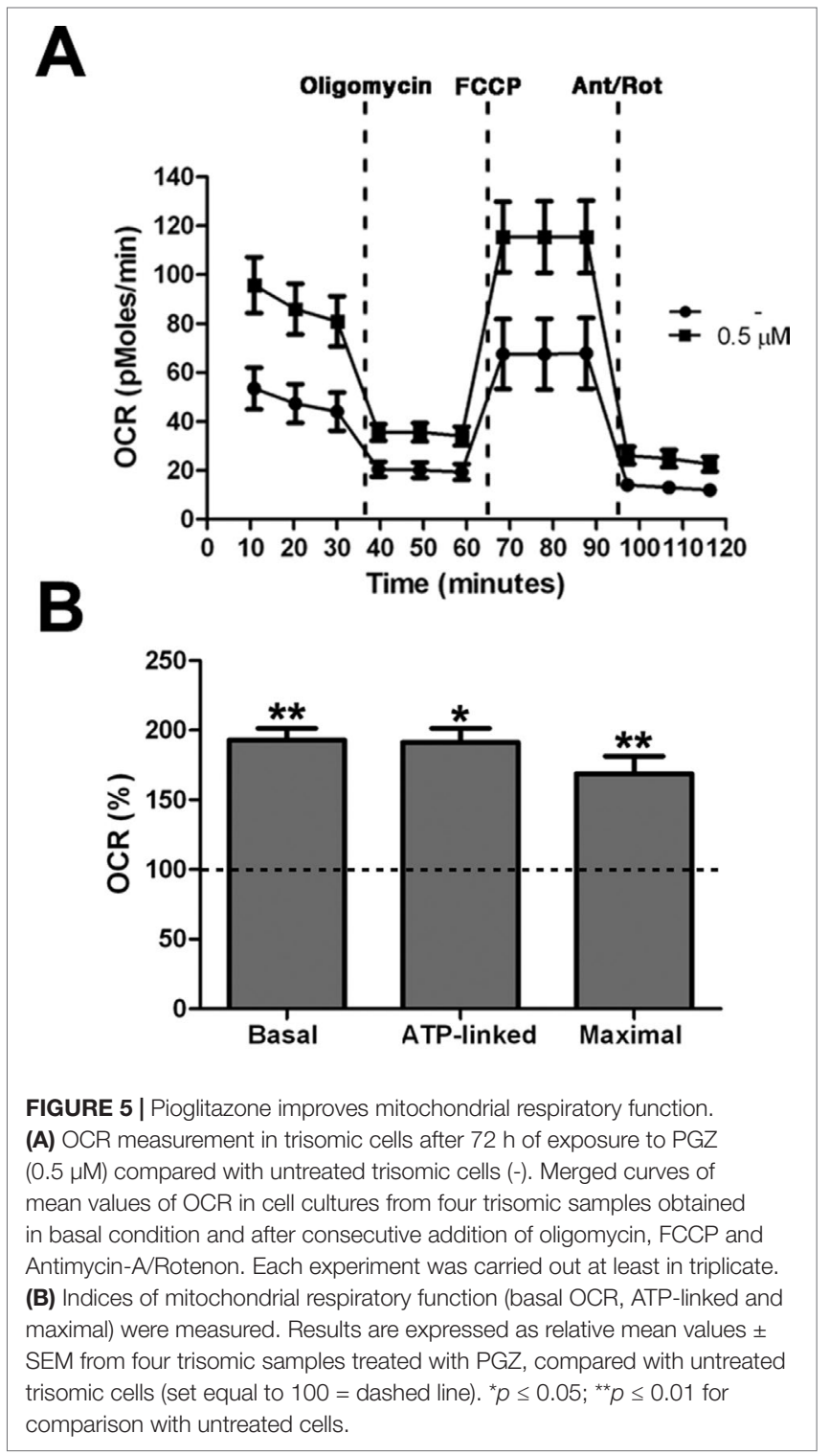

evidences support the hypothesis that targeting mitochondrial dysfunction in DS could help to counteract cognitive impairment and that pharmacologically induced transcriptional activation of the $P G C-1 \alpha$ pathway is expected to exert neuroprotective effects.

In a previous study we successfully tested the biguanide metformin as PGC-1 $\alpha$ activator and mitochondrial "benefactor" in a cell model of DS. Metformin enhanced OCR and ATP production promoting overall mitochondrial activity and biogenesis. The treatment also reversed the fragmentation of mitochondria observed in DS and increased the expression of genes of the fission/fusion machinery, namely OPA1 and MFN2 and caused a remodelling of mitochondrial cristae, thus correcting the anomalies observed by electron microscopy in trisomic cells (Izzo et al., 2017b). In the clinical practice, metformin is a well-tolerated drug but some side effects may occur (Krentz et al., 1994; Belcher et al., 2005), suggesting that alternative or synergistic possibilities may help to reduce even these mild side 
effects. For this reason, we have tested in a cell model of DS the effects of a thiazolinedione, namely PGZ, a PPAR- $\gamma$ agonist, which stimulates $P G C-1 \alpha$ expression. PGZ plays a neuroprotective role in dopaminergic neurons in both in vitro and in vivo PD models (Wang et al., 2017), which was associated with PGC-1 $\alpha$ induction and the regulation of proteins involved in mitochondria function (Wang et al., 2017). We intentionally used low doses of the drugs because both metformin and PGZ are known to inhibit respiratory complex I when used at high concentrations (Brunmair et al., 2004; Ghosh et al., 2007; Garcia-Ruiz et al., 2013). Therefore, we tested the effects of doses that are possibly below those inhibiting the respiratory chain, as we previously did for metformin. These doses demonstrated to be non-toxic at all, as they did not affect cell viability in DS-HFFs exposed for $72 \mathrm{~h}$ to the drug and to enhance both expression and activity of PGC-1 $\alpha$ in DS-HFFs. The data are compatible with the concept that the activation of PPAR- $\gamma$ by PGZ induces PGC-1 $\alpha$ expression (Villena, 2015).

Mitochondria are dynamic organelles, which readily adapt to changes in cellular energy demands through network remodeling by continuous fusion and fission processes (Benard et al., 2007; Mishra and Chan, 2016). Mitochondrial fusion results in the formation of an interconnected mitochondrial network that allows the mixing and redistribution of proteins and mtDNA (Nunnari et al., 1997). In contrast, mitochondrial fission leads to mitochondria with a fragmented morphology, which facilitates the segregation of damaged mitochondria (Berman et al., 2008; Lee and Yoon, 2016). Regulating the equilibrium between mitochondrial fusion and fission is essential to maintain mitochondrial integrity and function through the balance between mitochondrial biogenesis and degradation. These processes are intricately linked to changes in network morphology and spatio-temporal positioning of mitochondria and to their function (Hodneland Nilsson et al., 2015). They are essential for neuronal processes such as synaptogenesis, $\mathrm{Ca}^{2+}$ buffering, axonal transport, and bioenergetics (Oettinghaus et al., 2016).

We previously demonstrated that DS-HFFs display a mitochondrial network more fragmented with a higher number of short and globular mitochondria and a smaller average mitochondrial volume when compared with euploid controls (Izzo et al., 2017b). Mitochondrial morphology is crucial for cellular physiology, as changes in mitochondrial shape have been linked to neurodegeneration, lifespan and cell death and highlights the importance of targeting therapeutically mitochondrial dynamics (Campello and Scorrano, 2010). In the present study, trisomic cells treated by PGZ showed a network promotion and a near complete rescue of mitochondrial morphology. Analysis of genes involved in fission and fusion processes demonstrated that fusion inducing genes, downregulated in DS-HFFs, were increased by PGZ administration, while the fission inducing DRP1 gene, over-expressed in trisomic cells (Valenti et al., 2017), was downregulated by PGZ treatment. Protein expression of this gene was too variable and yielded no statistical significance thus indicating that post-transcriptional regulating events may have occurred in the time frame that we have tested. PGZ increased fusion protein Opa1 and Mfn2 expressions and decreased fission protein Drp1 expression also in the remnant kidney of nephrectomized rats (Sun et al., 2017). A relationship between $P G C-1 \alpha$ expression and mitochondrial fusion genes has been previously reported (Soriano et al., 2006; Rai et al., 2014) and silencing of $P G C-1 \alpha$ in smooth muscle cells is known to increase mitochondrial fragmentation (Ryan et al., 2013). These results, together with the rescue of mitochondrial morphology observed in treated cells, can be considered a proof that mitochondrial dynamics, altered in trisomic cells, is restored by the treatment.

The functional purpose of mitochondrial biogenesis is to maintain mitochondrial quality and secure sufficient ATP production. We previously demonstrated in trisomic cells decreased ATP production, reduced oxygen consumption, increased ROS production and increased levels of intra-mitochondrial calcium (Piccoli et al., 2013). PGZ treatment induced a recovery of mitochondrial respiration in DS-HFFs. In agreement with this finding, the direct measure of the basal ATP content demonstrated that PGZ treatment was associated to a significant increase of ATP in treated DS-HFFs. We speculate that this increase may be also affected by the increase of the adenine nucleotide translocators ANT1/SLC25A4 and ANT2/SLC25A5, previously found upregulated in response to $P G C-1 \alpha$ induction (Izzo et al., 2014). An inverse relationship between ANT1/SLC25A4 and the Hsa21 miRNA let-7c, which is upregulated in trisomic cells, has also been demonstrated (Izzo et al., 2017a). These data, together with a significant decrease of ROS production, provide strong evidence of a global improvement in energy metabolism and proof that PGZ is able to improve mitochondrial activity even at concentrations as low as $0.5 \mu \mathrm{M}$. This is important because higher doses deal with the risk of starting to inhibit mitochondrial complex I, as discussed above.

The complex neurological function is the result of many molecular, cellular and environmental events that are either initiated or completed before birth and must be coordinated at precisely the right time (Bartesaghi et al., 2015). This suggests that the overall impact of the neurological deficits in DS may be lessened if the initial pathologic changes in the brain are prevented from occurring. Permanent brain alterations of DS originate during fetal life in which the first window of opportunity for cognitive improvement occurs. Early prenatal diagnosis offers a temporal window to have a positive impact on brain development and to improve postnatal cognitive outcome in affected individuals. Only a few approaches (Epigallocatechine gallate, NAP/SAL, fluoxetine, and apigenin) have been used to treat mice in utero; some of these showed therapeutic effects that persisted to adulthood (Nakano-Kobayashi et al., 2017). Unfortunately, there is not enough information about safety of PGZ administration during pregnancy, even though no fetal malformation has been reported in the few monitored cases. Poor or discordant outcomes, controversial results of preclinical evaluations in mice and different degrees of rescue of neurogenesis obtained with previously tested antioxidants and nutraceutics (Izzo et al., 2018) suggest that combination of drugs may be both necessary and advantageous. Drug combinations offer potentially higher efficacy with lower individual drug dosage and have been considered to be beneficial in the treatment and management of other chronic medical conditions (Taylor, 2004; Muayqil and Camicioli, 2012). Different combinations of PGZ and metformin are already available in the clinical practice to improve glycemic control in patients with type 2 diabetes, with side effects similar to those that occur in mono-therapy. However, we suppose that the doses we need to govern mitochondrial dysfunction in DS could be lower than those commonly used for diabetes therapy. Further experiments will be needed to assess the smallest effective doses in a combinatory drug therapy. 


\section{DATA AVAILABILITY STATEMENT}

All datasets generated for this study are included in the manuscript and the supplementary files.

\section{ETHICS STATEMENT}

Human samples utilized in this study were obtained from the "Telethon Bank of Fetal Biological Samples," at the University of Naples Federico II, whose activities were approved by the local Institutional Ethics Committee in November 2001.

\section{AUTHOR CONTRIBUTIONS}

NM, AI, SP, and AC designed the research and wrote the manuscript. NM, MN, LZ, DF, TM, RA, and TP contributed to the methodology. FB and VS conducted the statistical analysis. AS, GC, RC, and RG contributed to the data curation. GM,

\section{REFERENCES}

Bartesaghi, R., Haydar, T. F., Delabar, J. M., Dierssen, M., Martinez-Cue, C., and Bianchi, D. W. (2015). New perspectives for the rescue of cognitive disability in down syndrome. J. Neurosci. 35, 13843-13852. doi: 10.1523/ JNEUROSCI.2775-15.2015

Beckervordersandforth, R., Ebert, B., Schaffner, I., Moss, J., Fiebig, C., Shin, J., et al. (2017). Role of mitochondrial metabolism in the control of early lineage progression and aging phenotypes in adult hippocampal neurogenesis. Neuron 93, 560-573. doi: 10.1016/j.neuron.2017.03.008

Belcher, G., Lambert, C., Edwards, G., Urquhart, R., and Matthews, D. R. (2005). Safety and tolerability of pioglitazone, metformin, and gliclazide in the treatment of type 2 diabetes. Diabetes Res. Clin. Pract. 70, 53-62. doi: 10.1016/j. diabres.2005.02.011

Benard, G., Bellance, N., James, D., Parrone, P., Fernandez, H., Letellier, T., et al. (2007). Mitochondrial bioenergetics and structural network organization. J. Cell. Sci. 120, 838-848. doi: 10.1242 /jcs. 03381

Berman, S. B., Pineda, F. J., and Hardwick, J. M. (2008). Mitochondrial fission and fusion dynamics: the long and short of it. Cell Death Differ. 15, 1147-1152. doi: $10.1038 /$ cdd.2008.57

Bogacka, I., Xie, H., Bray, G. A., and Smith, S. R. (2005). Pioglitazone induces mitochondrial biogenesis in human subcutaneous adipose tissue in vivo. Diabetes 54, 1392-1399. doi: 10.2337/diabetes.54.5.1392

Breidert, T., Callebert, J., Heneka, M. T., Landreth, G., Launay, J. M., and Hirsch, E. C. (2002). Protective action of the peroxisome proliferator-activated receptor-gamma agonist pioglitazone in a mouse model of Parkinson's disease. J. Neurochem. 82, 615-624. doi: 10.1046/j.1471-4159.2002.00990.x

Brunmair, B., Staniek, K., Gras, F., Scharf, N., Althaym, A., Clara, R., et al. (2004). Thiazolidinediones, like metformin, inhibit respiratory complex I: a common mechanism contributing to their antidiabetic actions? Diabetes 53, 1052-1059. doi: $10.2337 /$ diabetes.53.4.1052

Campello, S., and Scorrano, L. (2010). Mitochondrial shape changes: orchestrating cell pathophysiology. EMBO Rep. 11, 678-684. doi: 10.1038/embor.2010.115

Chaturvedi, R. K., and Beal, M. F. (2008). PPAR: a therapeutic target in Parkinson's disease. J. Neurochem. 106, 506-518. doi: 10.1111/j.1471-4159.2008.05388.x

Cheng, A., Wan, R., Yang, J. L., Kamimura, N., Son, T. G., Ouyang, X., et al. (2012). Involvement of PGC-1alpha in the formation and maintenance of neuronal dendritic spines. Nat. Commun. 3, 1250. doi: 10.1038/ncomms2238

Coletta, D. K., Sriwijitkamol, A., Wajcberg, E., Tantiwong, P., Li, M., Prentki, M., et al. (2009). Pioglitazone stimulates AMP-activated protein kinase signalling and increases the expression of genes involved in adiponectin signalling, mitochondrial function and fat oxidation in human skeletal
PP, and LN contributed to the funding acquisition and project administration. AI, AC, and $\mathrm{LN}$ supervised the research and revised the manuscript.

\section{FUNDING}

LN was supported by grants POR Campania FSE 2007-2013 Project CREME from Campania Region, POR Campania FSE 2014-2020 from Campania Region. GM was supported by grants from European Research Council Grant "menTORingTregs" n. 310496, Fondazione Italiana Sclerosi Multipla (FISM) n. 2016/R/18 and Telethon n. GGP17086.

\section{SUPPLEMENTARY MATERIAL}

The Supplementary Material for this article can be found online at: https:/www.frontiersin.org/articles/10.3389/fgene.2019.00606/ full\#supplementary-material muscle in vivo: a randomised trial. Diabetologia 52, 723-732. doi: 10.1007/ s00125-008-1256-9

Conti, A., Fabbrini, F., D’agostino, P., Negri, R., Greco, D., Genesio, R., et al. (2007). Altered expression of mitochondrial and extracellular matrix genes in the heart of human fetuses with chromosome 21 trisomy. BMC Genom. 8, 268. doi: 10.1186/1471-2164-8-268

Corona, J. C., and Duchen, M. R. (2015). PPARgamma and PGC-1alpha as therapeutic targets in Parkinson's. Neurochem. Res. 40, 308-316. doi: 10.1007/ s11064-014-1377-0

Corona, J. C., and Duchen, M. R. (2016). PPARgamma as a therapeutic target to rescue mitochondrial function in neurological disease. Free Radic. Biol. Med. 100, 153-163. doi: 10.1016/j.freeradbiomed.2016.06.023

Correia, S. C., and Moreira, P. I. (2018). "Role of Mitochondria in Neurodegenerative Diseases - The Dark Side of the "Energy Factory" " in Mitochondrial biology and experimental therapeutics. Ed. P. J. Oliveira (Cham, Switzerland: Springer). doi: 10.1007/978-3-319-73344-9 11

Coskun, P. E., and Busciglio, J. (2012). Oxidative stress and mitochondrial dysfunction in Down's syndrome: relevance to aging and dementia. Curr. Gerontol. Geriatr. Res. 2012, 383170. doi: 10.1155/2012/383170

Dong, W., Gao, D., and Zhang, X. (2007). Mitochondria biogenesis induced by resveratrol against brain ischemic stroke. Med. Hypotheses 69, 700-701. doi: 10.1016/j.mehy.2007.01.017

Escribano, L., Simon, A. M., Perez-Mediavilla, A., Salazar-Colocho, P., Del Rio, J., and Frechilla, D. (2009). Rosiglitazone reverses memory decline and hippocampal glucocorticoid receptor down-regulation in an Alzheimer's disease mouse model. Biochem. Biophys. Res. Commun. 379, 406-410. doi: 10.1016/j.bbrc. 2008.12.071

Fujisawa, K., Nishikawa, T., Kukidome, D., Imoto, K., Yamashiro, T., Motoshima, H., et al. (2009). TZDs reduce mitochondrial ROS production and enhance mitochondrial biogenesis. Biochem. Biophys. Res. Commun. 379, 43-48. doi: 10.1016/j.bbrc.2008.11.141

Garcia-Bueno, B., Caso, J. R., Perez-Nievas, B. G., Lorenzo, P., and Leza, J. C. (2007). Effects of peroxisome proliferator-activated receptor gamma agonists on brain glucose and glutamate transporters after stress in rats. Neuropsychopharmacology 32, 1251-1260. doi: 10.1038/sj.npp.1301252

Garcia-Ruiz, I., Solis-Munoz, P., Fernandez-Moreira, D., Munoz-Yague, T., and Solis-Herruzo, J. A. (2013). Pioglitazone leads to an inactivation and disassembly of complex I of the mitochondrial respiratory chain. BMC Biol. 11, 88. doi: 10.1186/1741-7007-11-88

Gardiner, K. J. (2015). Pharmacological approaches to improving cognitive function in Down syndrome: current status and considerations. Drug Des. Devel. Ther. 9, 103-125. doi: 10.2147/DDDT.S51476 
Genesio, R., Melis, D., Gatto, S., Izzo, A., Ronga, V., Cappuccio, G., et al. (2011). Variegated silencing through epigenetic modifications of a large $\mathrm{Xq}$ region in a case of balanced X;2 translocation with Incontinentia Pigmenti-like phenotype. Epigenetics 6, 1242-1247. doi: 10.4161/epi.6.10.17698

Ghosh, S., Patel, N., Rahn, D., Mcallister, J., Sadeghi, S., Horwitz, G., et al. (2007). The thiazolidinedione pioglitazone alters mitochondrial function in human neuron-like cells. Mol. Pharmacol. 71, 1695-1702. doi: 10.1124/mol.106.033845

Guidi, S., Bonasoni, P., Ceccarelli, C., Santini, D., Gualtieri, F., Ciani, E., et al. (2008). Neurogenesis impairment and increased cell death reduce total neuron number in the hippocampal region of fetuses with Down syndrome. Brain Pathol. 18, 180-197. doi: 10.1111/j.1750-3639.2007.00113.x

Guidi, S., Ciani, E., Bonasoni, P., Santini, D., and Bartesaghi, R. (2011). Widespread proliferation impairment and hypocellularity in the cerebellum of fetuses with Down syndrome. Brain Pathol. 21, 361-373. doi: 10.1111/j.1750-3639.2010. 00459.x

Guidi, S., Giacomini, A., Stagni, F., Emili, M., Uguagliati, B., Bonasoni, M. P., et al. (2018). Abnormal development of the inferior temporal region in fetuses with Down syndrome. Brain Pathol. 28, 986-998. doi: 10.1111/bpa.12605

Guihard-Costa, A. M., Khung, S., Delbecque, K., Menez, F., and Delezoide, A. L. (2006). Biometry of face and brain in fetuses with trisomy 21. Pediatr. Res. 59, 33-38. doi: 10.1203/01.pdr.0000190580.88391.9a

Hodneland Nilsson, L. I., Nitschke Pettersen, I. K., Nikolaisen, J., Micklem, D., Avsnes Dale, H., Vatne Rosland, G., et al. (2015). A new live-cell reporter strategy to simultaneously monitor mitochondrial biogenesis and morphology. Sci. Rep. 5, 17217. doi: 10.1038/srep 17217

Hofer, A., Noe, N., Tischner, C., Kladt, N., Lellek, V., Schauss, A., et al. (2014). Defining the action spectrum of potential PGC-1alpha activators on a mitochondrial and cellular level in vivo. Hum. Mol. Genet. 23, 2400-2415. doi: 10.1093/hmg/ddt631

Hunter, R. L., Choi, D. Y., Ross, S. A., and Bing, G. (2008). Protective properties afforded by pioglitazone against intrastriatal LPS in Sprague-Dawley rats. Neurosci. Lett. 432, 198-201. doi: 10.1016/j.neulet.2007.12.019

Izzo, A., Manco, R., Bonfiglio, F., Cali, G., De Cristofaro, T., Patergnani, S., et al. (2014). NRIP1/RIP140 siRNA-mediated attenuation counteracts mitochondrial dysfunction in Down syndrome. Hum. Mol. Genet. 23, 44064419. doi: $10.1093 / \mathrm{hmg} / \mathrm{ddu} 157$

Izzo, A., Manco, R., De Cristofaro, T., Bonfiglio, F., Cicatiello, R., Mollo, N., et al. (2017a). Overexpression of chromosome 21 miRNAs may affect mitochondrial function in the hearts of Down syndrome fetuses. Int. J. Genom. 2017, 8737649. doi: 10.1155/2017/8737649

Izzo, A., Mollo, N., Nitti, M., Paladino, S., Cali, G., Genesio, R., et al. (2018). Mitochondrial dysfunction in down syndrome: molecular mechanisms and therapeutic targets. Mol. Med. 24, 2. doi: 10.1186/s10020-018-0004-y

Izzo, A., Nitti, M., Mollo, N., Paladino, S., Procaccini, C., Faicchia, D., et al. (2017b). Metformin restores the mitochondrial network and reverses mitochondrial dysfunction in Down syndrome cells. Hum. Mol. Genet. 26, 1056-1069. doi: 10.1093/hmg/ddx016

Johri, A., Calingasan, N. Y., Hennessey, T. M., Sharma, A., Yang, L., Wille, E., et al. (2012). Pharmacologic activation of mitochondrial biogenesis exerts widespread beneficial effects in a transgenic mouse model of Huntington's disease. Hum. Mol. Genet. 21, 1124-1137. doi: 10.1093/hmg/ddr541

Khacho, M., and Slack, R. S. (2018). Mitochondrial dynamics in the regulation of neurogenesis: from development to the adult brain. Dev. Dyn. 247, 47-53. doi: 10.1002/dvdy. 24538

Krentz, A. J., Ferner, R. E., and Bailey, C. J. (1994). Comparative tolerability profiles of oral antidiabetic agents. Drug Saf. 11, 223-241. doi: 10.2165/ 00002018-199411040-00002

Lagouge, M., Argmann, C., Gerhart-Hines, Z., Meziane, H., Lerin, C., Daussin, F., et al. (2006). Resveratrol improves mitochondrial function and protects against metabolic disease by activating SIRT1 and PGC-1alpha. Cell 127, 1109-1122. doi: 10.1016/j.cell.2006.11.013

Landreth, G., Jiang, Q., Mandrekar, S., and Heneka, M. (2008). PPARgamma agonists as therapeutics for the treatment of Alzheimer's disease. Neurotherapeutics 5, 481-489. doi: 10.1016/j.nurt.2008.05.003

Lee, H., and Yoon, Y. (2016). Mitochondrial fission and fusion. Biochem. Soc. Trans. 44, 1725-1735. doi: 10.1042/BST20160129

Leone, T. C., Lehman, J. J., Finck, B. N., Schaeffer, P. J., Wende, A. R., Boudina, S., et al. (2005). PGC-1alpha deficiency causes multi-system energy metabolic derangements: muscle dysfunction, abnormal weight control and hepatic steatosis. PLoS Biol. 3, e101. doi: 10.1371/journal.pbio.0030101

Michalik, L., Auwerx, J., Berger, J. P., Chatterjee, V. K., Glass, C. K., Gonzalez, F. J., et al. (2006). International union of pharmacology. LXI. Peroxisome proliferator-activated receptors. Pharmacol. Rev. 58, 726-741. doi: 10.1124/ pr.58.4.5

Mishra, P., and Chan, D. C. (2016). Metabolic regulation of mitochondrial dynamics. J. Cell. Biol. 212, 379-387. doi: 10.1083/jcb.201511036

Mosmann, T. (1983). Rapid colorimetric assay for cellular growth and survival: application to proliferation and cytotoxicity assays. J. Immunol. Methods 65, 55-63. doi: 10.1016/0022-1759(83)90303-4

Muayqil, T., and Camicioli, R. (2012). Systematic review and meta-analysis of combination therapy with cholinesterase inhibitors and memantine in Alzheimer's disease and other dementias. Dement. Geriatr. Cogn. Dis. Extra 2, 546-572. doi: 10.1159/000343479

Nakano-Kobayashi, A., Awaya, T., Kii, I., Sumida, Y., Okuno, Y., Yoshida, S., et al. (2017). Prenatal neurogenesis induction therapy normalizes brain structure and function in Down syndrome mice. Proc. Natl. Acad. Sci. U. S. A. 114, 10268-10273. doi: 10.1073/pnas.1704143114

Nicolakakis, N., Aboulkassim, T., Ongali, B., Lecrux, C., Fernandes, P., RosaNeto, P., et al. (2008). Complete rescue of cerebrovascular function in aged Alzheimer's disease transgenic mice by antioxidants and pioglitazone, a peroxisome proliferator-activated receptor gamma agonist. J. Neurosci. 28, 9287-9296. doi: 10.1523/JNEUROSCI.3348-08.2008

Nunnari, J., Marshall, W. F., Straight, A., Murray, A., Sedat, J. W., and Walter, P. (1997). Mitochondrial transmission during mating in Saccharomyces cerevisiae is determined by mitochondrial fusion and fission and the intramitochondrial segregation of mitochondrial DNA. Mol. Biol. Cell. 8, 1233-1242. doi: 10.1091/ mbc.8.7.1233

Oettinghaus, B., Schulz, J. M., Restelli, L. M., Licci, M., Savoia, C., Schmidt, A., et al. (2016). Synaptic dysfunction, memory deficits and hippocampal atrophy due to ablation of mitochondrial fission in adult forebrain neurons. Cell Death Differ. 23, 18-28. doi: 10.1038/cdd.2015.39

Petrozzi, L., Ricci, G., Giglioli, N. J., Siciliano, G., and Mancuso, M. (2007). Mitochondria and neurodegeneration. Biosci. Rep. 27, 87-104. doi: 10.1007/ s10540-007-9038-Z

Petrozziello, T., Secondo, A., Tedeschi, V., Esposito, A., Sisalli, M., Scorziello, A., et al. (2017). ApoSOD1 lacking dismutase activity neuroprotects motor neurons exposed to beta-methylamino-L-alanine through the $\mathrm{Ca}(2+) / \mathrm{Akt} /$ ERK1/2 prosurvival pathway. Cell Death Differ. 24, 511-522. doi: 10.1038/ cdd.2016.154

Piccoli, C., Izzo, A., Scrima, R., Bonfiglio, F., Manco, R., Negri, R., et al. (2013). Chronic pro-oxidative state and mitochondrial dysfunctions are more pronounced in fibroblasts from Down syndrome foeti with congenital heart defects. Hum. Mol. Genet. 22, 1218-1232. doi: 10.1093/hmg/dds529

Porcelli, A. M., Pinton, P., Ainscow, E. K., Chiesa, A., Rugolo, M., Rutter, G. A., et al. (2001). Targeting of reporter molecules to mitochondria to measure calcium, ATP, and pH. Methods Cell Biol. 65, 353-380. doi: 10.1016/ S0091-679X(01)65021-0

Rai, M., Katti, P., and Nongthomba, U. (2014). Drosophila Erect wing (Ewg) controls mitochondrial fusion during muscle growth and maintenance by regulation of the Opal-like gene. J. Cell. Sci. 127, 191-203. doi: 10.1242/ jcs. 135525

Ryan, J. J., Marsboom, G., Fang, Y. H., Toth, P. T., Morrow, E., Luo, N., et al. (2013). PGC1alpha-mediated mitofusin-2 deficiency in female rats and humans with pulmonary arterial hypertension. Am. J. Respir. Crit. Care Med. 187, 865-878. doi: 10.1164/rccm.201209-1687OC

Sakamoto, J., Kimura, H., Moriyama, S., Odaka, H., Momose, Y., Sugiyama, Y., et al. (2000). Activation of human peroxisome proliferator-activated receptor (PPAR) subtypes by pioglitazone. Biochem. Biophys. Res. Commun. 278, 704711. doi: 10.1006/bbrc.2000.3868

Sauerbeck, A., Gao, J., Readnower, R., Liu, M., Pauly, J. R., Bing, G., et al. (2011). Pioglitazone attenuates mitochondrial dysfunction, cognitive impairment, cortical tissue loss, and inflammation following traumatic brain injury. Exp. Neurol. 227, 128-135. doi: 10.1016/j.expneurol.2010.10.003

Scarpulla, R. C., Vega, R. B., and Kelly, D. P. (2012). Transcriptional integration of mitochondrial biogenesis. Trends Endocrinol. Metab. 23, 459-466. doi: 10.1016/j.tem.2012.06.006 
Schindelin, J., Arganda-Carreras, I., Frise, E., Kaynig, V., Longair, M., Pietzsch, T., et al. (2012). Fiji: an open-source platform for biological-image analysis. Nat. Methods 9, 676-682. doi: 10.1038/nmeth.2019

Soriano, F. X., Liesa, M., Bach, D., Chan, D. C., Palacin, M., and Zorzano, A. (2006). Evidence for a mitochondrial regulatory pathway defined by peroxisome proliferator-activated receptor-gamma coactivator-1 alpha, estrogen-related receptor-alpha, and mitofusin 2. Diabetes 55, 1783-1791. doi: 10.2337/ db05-0509

Sun, L., Yuan, Q., Xu, T., Yao, L., Feng, J., Ma, J., et al. (2017). Pioglitazone improves mitochondrial function in the remnant kidney and protects against renal fibrosis in 5/6 nephrectomized rats. Front Pharmacol. 8, 545. doi: 10.3389/ fphar.2017.00545

Taylor, A. A. (2004). Combination drug treatment of hypertension: have we come full circle? Curr. Cardiol. Rep. 6, 421-426. doi: 10.1007/s11886-0040049-0

Torres, M. D., Garcia, O., Tang, C., and Busciglio, J. (2018). Dendritic spine pathology and thrombospondin-1 deficits in Down syndrome. Free Radic. Biol. Med. 114, 10-14. doi: 10.1016/j.freeradbiomed.2017.09.025

Valenti, D., Rossi, L., Marzulli, D., Bellomo, F., De Rasmo, D., Signorile, A., et al. (2017). Inhibition of Drp1-mediated mitochondrial fission improves mitochondrial dynamics and bioenergetics stimulating neurogenesis in hippocampal progenitor cells from a Down syndrome mouse model. Biochim. Biophys. Acta Mol. Basis Dis. 1863, 3117-3127. doi: 10.1016/j.bbadis.2017.09.014
Villena, J. A. (2015). New insights into PGC-1 coactivators: redefining their role in the regulation of mitochondrial function and beyond. FEBS J. 282, 647-672. doi: 10.1111 /febs. 13175

Wang, Y., Zhao, W., Li, G., Chen, J., Guan, X., Chen, X., et al. (2017). Neuroprotective effect and mechanism of thiazolidinedione on dopaminergic neurons in vivo and in vitro in Parkinson's disease. PPAR Res. 2017, 4089214. doi: $10.1155 / 2017 / 4089214$

Willson, T. M., Brown, P. J., Sternbach, D. D., and Henke, B. R. (2000). The PPARs: from orphan receptors to drug discovery. J. Med. Chem. 43, 527-550. doi: $10.1021 / \mathrm{jm} 990554 \mathrm{~g}$

Conflict of Interest Statement: The authors declare that the research was conducted in the absence of any commercial or financial relationships that could be construed as a potential conflict of interest.

Copyright (๑ 2019 Mollo, Nitti, Zerillo, Faicchia, Micillo, Accarino, Secondo, Petrozziello, Cali, Cicatiello, Bonfiglio, Sarnataro, Genesio, Izzo, Pinton, Matarese, Paladino, Conti and Nitsch. This is an open-access article distributed under the terms of the Creative Commons Attribution License (CC BY). The use, distribution or reproduction in other forums is permitted, provided the original author(s) and the copyright owner(s) are credited and that the original publication in this journal is cited, in accordance with accepted academic practice. No use, distribution or reproduction is permitted which does not comply with these terms. 